\title{
Advances in Intermixed Quantum Well Devices
}

\author{
E. Herbert Li*, Senior Member, IEEE \\ Department of Electrical \& Electronic Engineering, University of Hong Kong, Pokfulam Road, Hong Kong
}

\begin{abstract}
Quantum well composition intermixing is a thermal induced interdiffusion of the constituent atoms through the hetero-interface. The intermixed structures created by both impurity induced and impurity free or vacancy promoted processes have recently attracted high attention. The interdiffusion mechanism is no longer confined to a single phase diffusion for two constituent atoms, but it can now consist of two or multiple phases and/or for multiple species, such as three cations interdiffusion and two pairs of cation-anion interdiffusion. A review on the impact of intermixing on device physics is presented with many interesting features. For instance, both compressive or tensile strain materials and both blue or red shifts in the bandgap can be achieved depending on the types of intermixing. The recent advancement in intermixing modified optical properties, such as absorption, refractive index as well as electro-optics effects are discussed. In addition, this paper will place a strong emphasis on the device application of the intermixing technology. The advantage of being able to tune the material provides a way to improve the performance of photodetectors and modulators. Attractive distributedfeedback and vertical cavity laser dynamics have been shown due to some unique device physics of the quantum well intermixing. Several state-of-the-art results will be summarized with an emphasis on its future development and directions.
\end{abstract}

\section{Introduction}

A practical technique for integrating active and passive photonic devices will produce an immense impact on information processing, distribution, and manipulation. It is expected to be as significant a technological advance as was the development of the electronic IC. However, despite substantial efforts made by a large number of groups worldwide over the last few years, and the increasing demand for multiwavelength optical networks generated by the exponential growth of the internet and the World Wide Web in particular, this utopian vision has yet to be realized, where a consequence of the magnitude of the technological challenges is involved. For practical photonic IC's, practicality mandates low manufacturing cost and high reliability (low operating cost) even at the expense of somewhat reduced performance. With this in mind, we can establish the most important requirement for monolithically integrating optoelectronic devices of

differing functionality's in order to achieve practical waveguiding photonic IC's. One of the primary requirements for this waveguiding is bandgap (operation wavelength) compatibility among the various optoelectronic devices. Although a number of techniques exist, each has its advantages and disadvantages - none is a panacea.

(a)

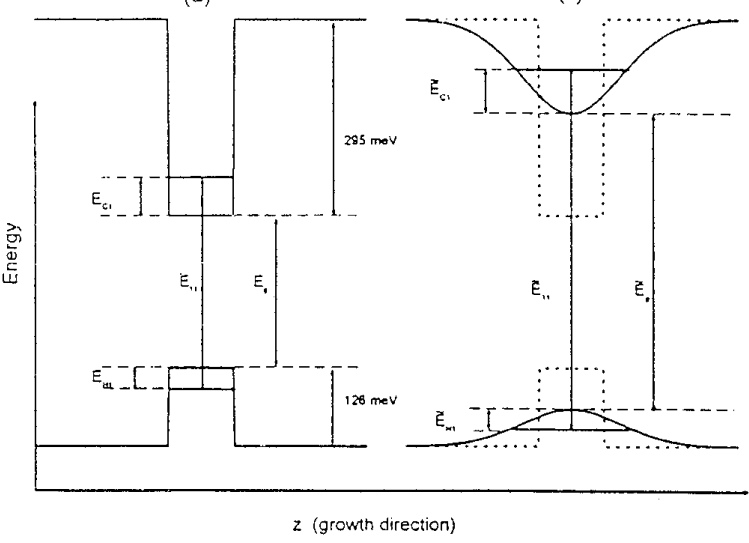

Figure 1. The conduction band and valence band potential energy profiles for an as-grown rectangular SQW structure and that of its interdiffused structure where $\mathrm{L}_{\mathrm{d}} \approx 1 / 2 \mathrm{~L}_{\mathrm{z}}$. The tilde denotes the respective parameter in an interdiffused SQW and $E_{c f}$ is the sum of the electron $E_{c 1}$ and hole $E_{h 1}$ confinement energies [1]

A simple technique of fabricating integrated optoelectronic devices is proposed using spatially selective modification of quantum well (QW) shape after standard growth which, in turn, modifies QW bandgap energy $[2,3]$. This diffused quantum well (DFQW) is a non-square quantum well produced by interdiffusion of constituent atoms through the heterointerface (Fig. 1). In the literature, DFQW is also referred to as quantum well mixing or intermixing (QWI) and quantum well disordering (IID). It is based on the fact that a $\mathrm{QW}$ is an inherently metastable system due to the large concentration gradient of atomic species across the well/barrier interface. For example, in InGaAs/InGaAsP QWs, the phosphorous concentration changes from $43 \%$ to $0 \%$ in a distance of less than $1 \mathrm{~nm}$. At temperature above $750^{\circ} \mathrm{C}$ significant diffusion of atomic species will occur resulting in an interdiffusion of the well and barrier alloys. This process causes an increase in the bandgap energy. It can also be greatly enhanced by the presence of impurities or defects or vacancies in the vicinity of the interfaces of the $\mathrm{QW}$, allowing interdiffusion

Email: ehli@eee.hku.hk 
to occur at temperatures that are substantially lower than that normally required. All these techniques are spatially selective, permitting interdiffusion enhancement only in the regions requiring a large bandgap, leaving other regions unmodified.

This bandgap modification is a powerful technique for monolithically integrating optoelectronic devices of varying functionalities on a single wafer. It is simple, has no deleterious effects on the optical, electrical, or lifetime properties of optoelectronic devices and, in fact, possesses surprising advantages (reducing polarization sensitivity and enhancing reflectance change). Since the process is easy to implement, it will simplify the fabrication of many complex photonic IC's and may finally render them practical enough to be adopted by industry. The advantage this technology is, through interdiffusion (intermixing), to accruate modify the QW materials (AlGaAs/GaAs, InGaAs/GaAs and InGaAs(P)/InP) bandgap and optical properties, and to make use of these tuning ability to integrate several device structures (such as waveguide-detector, laser-modulator, and laser-laser) to form photonic IC's. In particular, this allows a multisection integrated structure will be fabricated for wide band-width and multi-wavelengths applications, such as to demonstrate its use for wavelength division multiplexing (WDM) in high bit rate communication systems.

\section{Techniques for $\mathrm{QW}$ Intermixing}

\section{$2.1 \quad$ Ion Implantation}

Ion Implantation is a technique in which direct injection of ionized, energetic atoms or molecules into a solid is employed. The ions injected will carry energies ranging from a few $\mathrm{keV}$ to several $\mathrm{MeV}$, and implant doses from $10^{10}$ to more than $10^{16}$ ions $/ \mathrm{cm}^{2}$. Using small implantation energy, the damage introduced to the lattice structure will be reduced but with a decrease in the penetration depth, while with small dose of implants, there will be lesser enhancement of diffusion rate.

\subsection{Impurity Free Vacancy Diffusion (IFVD)}

The mechanism of IFVD requires the encapsulation of MQW samples by a dielectric cap such as $\mathrm{SiO}_{2}$ or $\mathrm{Si}_{3} \mathrm{~N}_{4}$ and then annealing at high temperature around $850{ }^{\circ} \mathrm{C} \sim 900{ }^{\circ} \mathrm{C}$ for 30 to 200 seconds. This will lead to out-diffusion of $\mathrm{Ga}$ into the cap and vacancies are generated on the group III sublattice that diffuse to the barriers and promote the interdiffusion in the MQWs. By using different combination of caps, selective area bandgap control is possible. The advantage of using IFVD is that it is a simple method which requires much less equipment to perform. IFVD can create large bandgap energy shifts without these disadvantages of IID. However, using IFVD will increase the number of etching steps needed to control the thickness of $\mathrm{SiO}_{2}$. Moreover, control of oxygen composition in $\mathrm{SiO}_{x} \mathrm{~N}_{y}$ is complex.

\subsection{Laser Assisted Disordering (LAD)}

Laser induced QW intermixing has been studied for some time, It is a direct write process that can pattern impurity induced layer disordering. This technique employs a highly focused $\mathrm{Ar}^{+}$laser beam. For fabrication of AlGaAs-GaAs DFQW, the laser beam with lasing wavelength of $488 \mathrm{~nm}$, is scanned through the heterostructure sample which is encapsulated with a $90 \mathrm{~nm}$ layer of $\mathrm{Si}_{-} \mathrm{Si}_{3} \mathrm{~N}_{4}$. Its scan speed can be as high as $85 \mu \mathrm{m} / \mathrm{s}$. The laser beam interaction region will result in a smooth cylindrical section on the micron scale. Annealing is then applied to drive the $\mathrm{Si}$ into the as-grown crystal, resulting in a local mixing of the crystal layers. Alternatively, pulsed photoabsorption-induced disordering (PAID) technique can be used to selectively intermix GaInAs/GaInAsP QW structure, which has been studied by the use of high spatial resolution time-resolved PL. Measurements showed that a reduction in the non-radioactive recombination time of nearly two orders of magnitude as a result of this intermixing technique.

\section{Band-gap Engineering}

Band gap modification in $\mathrm{Ne}^{+}$-ion implanted InGaAs/InP (for various $\mathrm{Ga}$ concentrations) and InAsP/InP (for $32 \% \mathrm{As}$ ) quantum well structures has been studied by low temperature PL spectra [16]. The maximum usable high temperature anneal for inducing the intermixing using an InP proximity cap is found to be $\sim 700$ degrees $\mathrm{C}$ for 13 s. A second low-temperature (300 degrees C) anneal, following the high-temperature (700 degrees $\mathrm{C}$ ) anneal, is found to induce greater band gap changes than the simple one-step anneal at 700 degrees $C$. The changes are found to be approximately proportional to the difference of bandgap energy between the well and the barrier materials; the proportionality coefficient increases with ion dose and reaches a maximum at a dose of $\sim 2 \times 10^{13} \mathrm{~cm}^{-2}$. Another study on the effects of defect-enhanced, impurity-free, quantum-well(QW)-barrier compositional intermixing caused by the $\mathrm{SiO}^{2}$ cap annealing at 750 degrees $\mathrm{C}$ of a 1.5- $\mu \mathrm{m}$ InGaAsP/InP MQW laser structure have been studied by PL [17]. A substantial band-gap blue shift, as much as $112 \mathrm{~nm}(\sim 66 \mathrm{meV})$, was found in the structure and the value of the shift can be controlled by the anneal time. The amount of the shift does not depend on the thickness of the $\mathrm{SiO}^{2}$ cap layer. The lasing wavelength of the laser produced with the $\mathrm{SiO}^{2}$ cap has a $78 \mathrm{~nm}$ blue shift over that of the laser without the cap. 


\section{Device Applications}

\subsection{Laser Diodes}

The effects of defect-enhanced, impurity-free, QW-barrier compositional intermixing caused by the $\mathrm{SiO}_{2}$ cap annealing at $750{ }^{\circ} \mathrm{C}$ of a $1.5 \mu \mathrm{m}$ InGaAsP/InP multiple quantum-well (MQW) laser structure have been studied by PL [4]. A substantial band-gap blue shift, as much as 112 $\mathrm{nm}(\sim 66 \mathrm{meV})$, was found in the structure and the value of the shift can be controlled by the anneal time. The amount of the shift does not depend on the thickness of the $\mathrm{SiO}_{2}$ cap layer. Ridge-waveguide lasers were fabricated on the different areas of the wafer, with and without a $\mathrm{SiO}_{2}$ cap during a $60 \mathrm{~s}$ anneal. The lasing wavelength of the laser produced with the $\mathrm{SiO}_{2}$ cap has a $78 \mathrm{~nm}$ blue shift over that of the laser without the $\mathrm{SiO}_{2}$ cap.

By using phosphorous doped (5\% wt P) silica as masking material and standard silica capping to promote QW interdiffusion, GaAs-AlGaAs ridge lasers with integrated transparent waveguides were fabricated [5]. With a selective differential blue-shift of $30 \mathrm{~nm}$ in the absorption edge, devices with $400-\mu \mathrm{m} / 2.73-\mathrm{mm}$-long active/passive sections exhibited threshold currents of 8 $\mathrm{mA}$ in $\mathrm{CW}$ operation, only $1 \mathrm{~mA}$ higher than that for normal lasers of the same active length and from the same chip. This $14 \%$ increase in threshold current was accompanied by a slope efficiency decrease of $40 \%$. Losses of $3.2 \mathrm{~cm}^{-1}$ were measured in the passive waveguides at the lasing wavelength using the Fabry-Perot resonance method. This value is among the lowest reported so far using an impurity-free disordering technique.

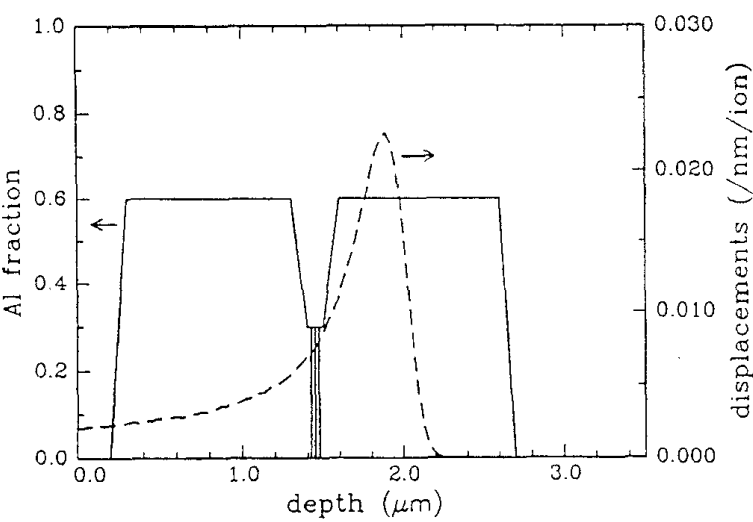

Figure 2. Schematic of the GRINSCH quantum well laser structure used in this study. Also shown is the calculated displacement profile for $240 \mathrm{keV}$ protons [6].

Reflectance modulation measurements have been used to determine facet temperatures of InGaAs/GaAs double-QW graded-index separate-confinement heterostructure ridge-waveguide lasers possessing band gap tuned passive cavity sections [7]. It is found that the incorporation of transparent extended cavities, produced by ion-implantation enhanced QW intermixing, significantly decreases the laser facet temperatures. The reduced photoabsorption occurring at the facets, achieved by the QW intermixing process, should lead to increases in both the maximum optical power levels and device longevity prior to the onset of catastrophic failure.

Proton irradiation followed by rapid thermal annealing was used to selectively induce layer intermixing and thus shift the emission wavelengths of GaAsAlGaAs graded-index separate-confinement-heterostructure QW lasers (Fig. 2) [6]. Up to $40 \mathrm{~nm}$ shifts were observed in 4 $\mu \mathrm{m}$ ridge waveguide devices irradiated to a dose of $1.5 \times$ $10^{16} \mathrm{~cm}^{-2}$. Although the wavelength shifts were accompanied by some degradation in the lasing threshold current and differential quantum efficiency, they were still quite acceptable at moderate wavelength shifts. This technique provides a simple and promising postgrowth process of integrating lasers of different wavelengths for wavelength-division-multiplexing applications.

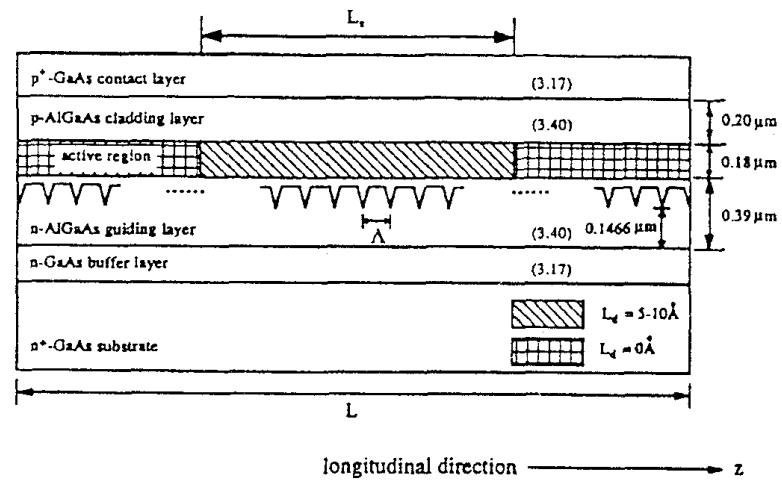

Figure 3. Schematic of the DFB laser with diffusion step profile. $L_{s}$ is the length of the diffusion step and $L_{d}$ is the diffusion length [9]

A two-dimensional self-consistent physical modeling has been employed to a particularly promising lateral current injection laser in order to gain insight into the physical mechanisms governing the operation of this family of devices [8]. Substantial benefits have been demonstrated from improved hole injection facilitated by relatively light p-type doping of barrier layers; from lateral shifting of the transverse junction to improve the overlap between the photon field and material gain; and from creating a lateral heterobarrier via $\mathrm{QW}$ intermixing in order to confine carriers in the lateral direction. It is found that with a number of relatively minor physically motivated modifications to existing fabrication processes, lateral injection lasers have the potential to exhibit greatly improved performance characteristics and to realize thereby their tremendous potential as enablers of 
optoelectronic integrated circuits and novel device structures.

DFB and Fabry-Perot (FP) semiconductor lasers with step (Fig. 3) and periodic interdiffusion quantum-well structures have been proposed for high-power singlelongitudinal-mode operation [9]. It is shown that the phaseadjustment region formed by the diffusion step (i.e., step change in optical gain and refractive index) counteracts the influence of spatial hole burning, especially for DFB lasers with large coupling-length products biased at high injection current. Furthermore, it is found that with careful design of the diffusion grating (i.e., grating period and amount of diffusion extent) of FP lasers, side-mode suppression ratio can be enhanced and threshold current density can be minimized to a satisfied level.

\subsection{Optical modulator and Detector}

A Fabry-Perot reflection-type modulator which uses interdiffused AlGaAs-GaAs QW as the active cavity material has been studied and optimized theoretically [10]. An asymmetric Bragg reflector structure (modeled by transfer matrices), with a doped depletion layer in the heterostructure, has been considered. Results show improvement in modulation property over its as-grown rectangular QW modulator. In particular, the change of reflectance in the diffused QW modulator is almost 0.6 to 0.7 , which is higher than that of the typically available values $(\sim 0.5$ to 0.6$)$, while the OFF-state on-resonance reflectance is almost close to zero. The operation voltage is also reduced by more than half as the interdiffusion becomes extensive. The finesse of the more extensively diffused quantum well also increases. Both of these features contribute to an improvement of the change of reflectance in the modulator. The operation wavelengths can be adjusted over a range of $100 \mathrm{~nm}$. However, the absorption coefficient change of the diffused quantum well increases only when there is a small amount of interdiffusion.

Waveguide phase modulators (Fig. 4), with 0.5 and $1-\mu \mathrm{m} Q W$ active regions which are defined by impurity-induced disordering are investigated theoretically [11]. By controlling the extent of the interdiffusion in the lateral claddings, the refractive index difference between the core and claddings is used to provide single-mode operation. Strong optical confinement, which is required to produce single-mode high-efficiency modulation, requires the peak impurity concentration to be at the center of the QW active region. Moreover, the annealing time needs to be optimized so that single mode can be maintained at the desired bias field. A low dopant concentration is also expected to minimize the destruction of the modulator structure. The results show that since the core/cladding interface is graded, the width of the metal contact is important. A comparison of modulation efficiency for active layer thicknesses of 0.5 and $1.0 \mu \mathrm{m}$ shows that the $0.5-\mu \mathrm{m}$ one is a more efficient structure and its absorption loss can be reduced by increasing the applied field from 50 to $100 \mathrm{kV} / \mathrm{cm}$.

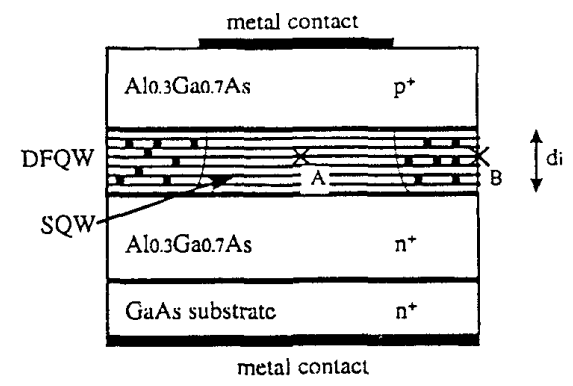

Figure 4. The cross section of the waveguide-type phase modulator, where the guiding region contains square QW's after interdiffusion [11].

The effect of RTA on important detector characteristics such as dark current, absolute response, noise, and detectivity is investigated for $\mathrm{QW}$ infrared photodetectors (QWIP) operating in the 8-12 $\mu \mathrm{m}$ wavelength regime [12]. A comprehensive set of experiments is conducted on QWIPs fabricated from both as-grown and annealed multiple-QW structures. RTA is done at an anneal temperature of 850 degrees $\mathrm{C}$ for $30 \mathrm{~s}$ using an $\mathrm{SiO}_{2}$ encapsulant. In general, a decrease in performance is observed for RTA QWIP when compared to the as- grown detectors. The peak absolute response of the annealed QWIPs is lower by almost a factor of four, which results in a factor of four decrease in quantum efficiency. In addition, a degraded noise performance results in a detectivity which is five times lower than that of QWIPs using as grown structures. Theoretical calculations of the absorption coefficient spectrum are in excellent agreement with the experimental data.

\subsection{Integrated Photonic Devices}

The bandgap of InGaAs-InGaAsP MQW material can be accurately tuned by photoabsorption-induced disordering (PAID), using a Nd:YAG laser, to allow lasers, modulators, and passive waveguides to be fabricated from a standard MQW structure [13]. The process relies on optical absorption in the active region of the MQW to produce sufficient heat to cause interdiffusion between the wells and barriers. Bandgap shifts larger than $100 \mathrm{meV}$ are obtainable using laser power densities of around $5 \mathrm{~W} \mathrm{~mm}^{-2}$ and periods of illumination of a few minutes to tens of minutes. This process provides an effective way of altering the emission wavelengths of lasers fabricated from a single 
epitaxial wafer. Blue shifts of up to $160 \mathrm{~nm}$ in the lasing spectra of both broad-area and ridge waveguide lasers have been achieved (Fig. 5). The bandgap-tuned lasers are assessed in terms of threshold current density, internal quantum efficiency, and internal losses. The ON/OFF ratios of bandgap-tuned electroabsorption modulators were tested over a range of wavelengths, with modulation depths of $20 \mathrm{~dB}$ obtained from material which has been bandgapshifted by $120 \mathrm{~nm}$, while samples shifted by $80 \mathrm{~nm}$ gave modulation depths as high as $27 \mathrm{~dB}$. Single-mode waveguide losses are as low as $5 \mathrm{~dB} \mathrm{~cm}^{-1}$ at $1550 \mathrm{~nm}$. Selective-area disordering has been used in the fabrication of extended cavity lasers. The retention of good electrical and optical properties in intermixed material demonstrates that PAID is a promising technique for the integration of devices to produce photonic integrated circuits.

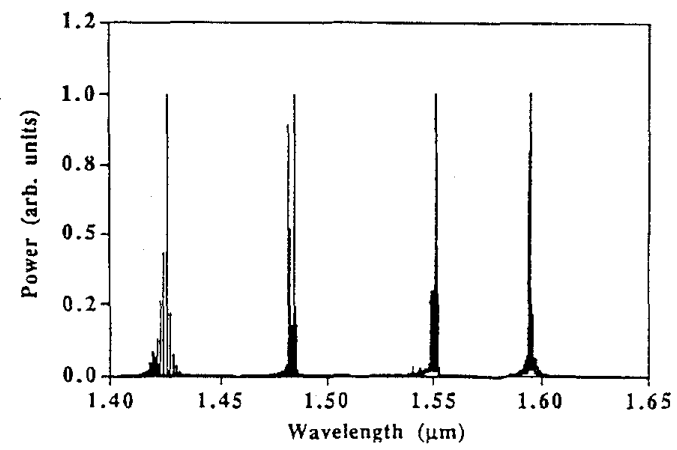

Figure 5. Emission spectra of bandgap-tuned oxide stripe lasers [13].

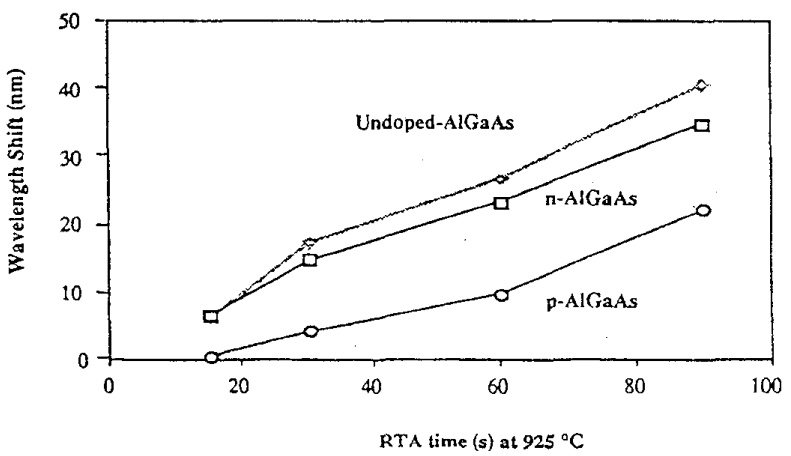

Figure 6. Wavelength shifts as a function of annealing time obtained from $\mathrm{SiO}_{2}$ capped intrinsic, n-I-o, and p-I-n samples annealed at $925^{\circ} \mathrm{C}$ [15].

By using the technique of QWI, monolithically integrated passive, and active waveguides can be fabricated [14]. It has been shown that mode-locked extended cavity semiconductor lasers with integrated low-loss passive waveguides display superior performance to devices in which the entire waveguide is active: the threshold current is a factor of 3-5 lower, the pulsewidth is reduced from $10.2 \mathrm{ps}$ in the all active laser to $3.5 \mathrm{ps}$ in the extended cavity device and there is a decrease in the free-running jitter level from 15 to $6 \mathrm{ps}(10 \mathrm{kHz}-10 \mathrm{Mhz})$.

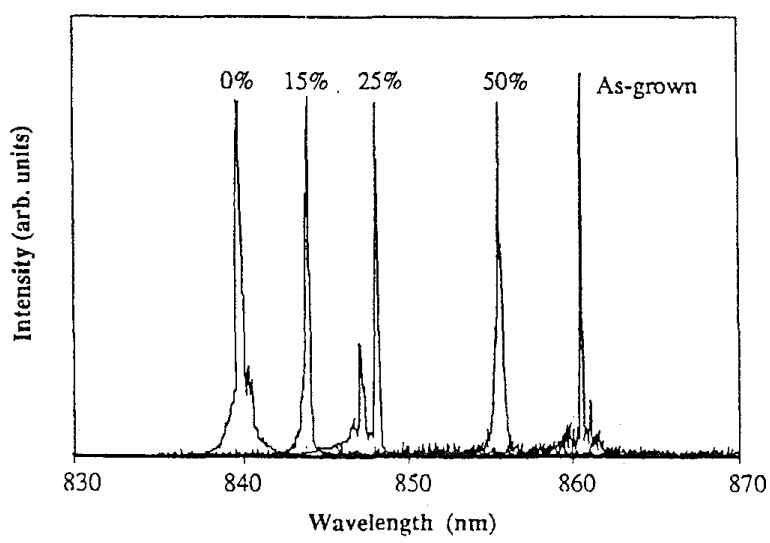

Figure 7. The lasing spectra of lasers bandgap-shifted using the SISA technique [15].

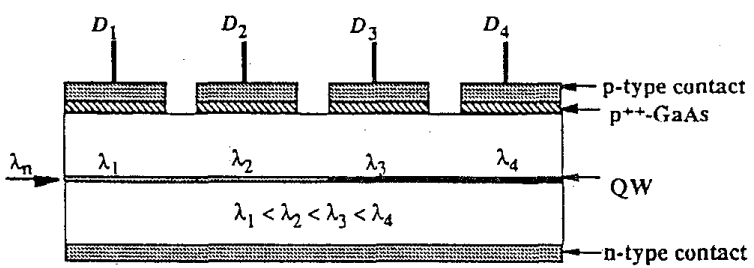

Figure 8. Schematic cross section of the four-channel demultiplexing wavefuide photodetector. The length of each diode is $300 \mathrm{~m}$ and the spacing between diodes is $100 \mathrm{~m}$. Light was injected from the cleaved edge of diode $\mathrm{D}_{1}$ during the photocurrent measurement [15].

Impurity-free vacancy disordering using $\mathrm{SiO}_{2}$ and $\mathrm{SrF}_{2}$ dielectric caps (Fig. 6) to induce selective quantumwell (QW) intermixing in the GaAs-AlGaAs system is studied [15]. The intermixing rate of IFVD was found to be higher in $n-i-p$ and intrinsic than in $p-i-n$ structures, which suggests that the diffusion of the Group III vacancy is not supported in p-type material. Single-mode waveguides have been fabricated from both as-grown and bandgaptuned double-quantum-well (DQW) laser samples. Propagation losses as low as $8.5 \mathrm{~dB} \mathrm{~cm}^{-1}$ were measured 
from the bandgap-tuned waveguides at the lasing wavelength of the undisordered material, i.e., $860 \mathrm{~nm}$. Simulation was also carried out to study the contribution of free-carrier absorption from the cladding layers, and the leakage loss induced by the heavily p-doped GaAs contact layer. It was found that the leakage loss contributed by the GaAs cap layer is significant and increases with wavelength. Based on IFVD, the fabrication of multiplewavelength lasers and multichannel wavelength division multiplexers were also demonstrated using the one-step "selective intermixing in selected area" technique. This technique enables one to control the degree of intermixing across a wafer. Lasers with bandgaps tuned to five different positions have been fabricated on a single chip (Fig. 7). These lasers showed only small changes in transparency current, internal quantum efficiency, or internal propagation loss, which indicates that the quality of the material remains high after being intermixed. Four-channel wavelength demultiplexers based on a waveguide photodetector design have also been fabricated (Fig. 8). Photocurrent and spontaneous emission spectra from individual diodes showed that the absorption edge was shifted by different degrees due to the selective degree of $\mathrm{QW}$ intermixing. The results obtained also imply that the technique can be used in the fabrication of broadwavelength emission superluminenscent diodes.

\section{Conclusion}

It can bee seen that QWI has introduced a very promising future in optoelectronics. In fact, development has advanced not only in improving performance, reliability, and integration of existing optical components, but also for cultivating novel optical functions. A planar, compatible photonic IC in which a variety of different optical devices are composed of a common as-grown MQW structure, is proposed. This integration technique does not require a re-growth process, and it should prove useful for a variety of integrated devices, including modelocked lasers and lasers integrated with optical modulators. At present, development for device enhancement and progressions is underway. In addition, wavelength demultiplexing modulator is foreseen as a new area of development in the very near future. Another aspect which is of interest will be towards the longer wavelengths (Mid to Far IR) using materials such as $\mathrm{InGaSb} / \mathrm{GaSb}$ as well as towards the shorter wavelengths (visible to UV range) using materials such as $\mathrm{InGaN} / \mathrm{GaN}$. These are now being developed and will expand the area of application by using QW intermixing.

\section{Acknowledgement}

The author is supported by RGC earmarked grant of Hong Kong and CRCG Research Grant of University of Hong Kong.

\section{Reference}

[1] P.J. Hughes, B.L. Weiss, and H.E. Jackson, Semicond. Sci. Technol. 12, 808 (1997).

[2] E.H.Li, Editor, Quantum Well Mixing and Optoelectronic Device Applications, Milestone Series, vol. 145, Bellingham, WA: SPIE Press, 1998.

[3] E.H.Li, Editor, Semiconductor Quantum Well Intermixing - Material Properties and Optoelectronics Applications, Series on Optoelectronic Properties of Semiconductors and Superlattices, Amsterdam: Gordon \& Breach, 1998.

[4] F.Camacho, E.A. Avrutin, P. Cusumano, A.S. Helmy, A.C. Bryce, and J.H. Marsh, IEEE Photon. Technol. Lett. 9, 1208 (1997).

[5] N.Cao, B.B. Elenkrig, J.G. Simmons, D.A. Thompson, and N. Puetz, Appl. Phys. Lett. 70, 3419 (1997).

[6] W.C.H.Choy and E.H.Li, IEEE J. Quantum Electron. 33, 382 (1997).

[7] W.C.H.Choy, B.L.Weiss and E.H.Li, IEEE J. Quantum Electron. 34, 84 (1998).

[8] P.Cusumano, J.H. Marsh, M.J. Rose, and J.S. Roberts, IEEE Photon. Techonol. Lett. 9, 282 (1997).

[9] A.McKee, C.J.. Mclean, G. Lullo, A.C. Bryce, R.M. Delarue, J.H. Marsh, and C.C. Button, IEEE J. Quantum Electron. 33, 45 (1997).

[10] B. S. Ooi, K. Mcllvaney, M. W. Street, A. Saher Helmy, S. G. Ayling, A. C. Bryce, J. H. Marsh, and J. S. Roberts, IEEE J. Quantum Electron. 33, 1784 (1997).

[11] P.G.Piva, S. Fafard, M. Dion, M. Buchanan, S. Charbonneau, R.D. Goldberg, and I.V. Mitchell, Appl. Phys. Lett. 70, 1662 (1997).

[12] E. H. Sargent, G. L. Tan, and J. M. Xu, IEEE JTSQE 3, 507 (1997).

[13] D.K.Sengupta, W. Fang, J.I. Malin, A.P. Curtis, T. Horton, H.C. Kuo, D. Turnbull, C.H. Lin, J. Li, K.C. Hsieh, S.L. Chuang, I. Adesida, M. Feng, S.G. Bishop, G.E. Stillman, J.M. Gibson, H. Chen, J. Mazumder, and H.C. Liu, J. Electron. Mat. 26, 43 (1997).

[14] H. H. Tan and C. Jagadish, Appl. Phys. Lett. 71, 2680 (1997).

[15] S.F.Yu, C.W.Lo, and E.H.Li, IEEE J. Quantum Electron. 33, 999 (1997).

[16] J.Z. Wan, J.G. Simmons, and D.A. Thompson, J. Appl. Phys. 81, 765 (1997).

[17] N. Cao, B.B. Elenkrig, J.G. Simmons, D.A. Thompson, and N. Puetz, Appl. Phys. Lett. 70, 3419 (1997). 